\title{
Exact Solutions for an Unsteady Flow of Viscoelastic Fluid in Cylindrical Domains Using the Fractional Maxwell Model
}

\author{
Kavita Khandelwal • Vatsala Mathur
}

Published online: 20 November 2014

(C) Springer India Pvt. Ltd. 2014

\begin{abstract}
This paper deals with the unsteady flow of an incompressible fractional Maxwell fluid filled in the annular region between two infinite coaxial circular cylinders. The motion of the fluid is due to the inner cylinder that applies a time dependent torsional shear to the fluid and outer cylinder which is moving at a constant velocity. The velocity field and shear stress are determined by the Laplace and finite Hankel transforms. The obtained solutions are presented in terms of the generalized $\mathrm{G}$ and R functions. Solutions for Ordinary Maxwell fluid and Newtonian fluid are also obtained by imposing appropriate limits. Finally, the influence of different values of parameters, constants and fractional coefficient, as well as a comparison between the velocity field and shear stress are also analyzed using graphical illustration.
\end{abstract}

Keywords Velocity field · Shear stress · Fractional calculus · Hankel transform · Laplace transform

\section{Introduction}

Study of the fluid motion in cylindrical domains is not only of fundamental theoretical interest but it also has many applications in the food industry, oil exploitation, chemistry and bioengineering.

The non-Newtonian fluids, such as lava, gums, slurries, emulsions, blood etc, are very frequently encountered in many different fields such as food industries, chemical engineering, biomedicine etc. and also are relevant to many other industrial processes. Hence, it is necessary to study the non-Newtonian fluid flows. Typical non-Newtonian characteristics include shear thinning, viscoelasticity, viscoplasticity and shear thickening behavior. Because of these complex behaviors, there are various models suggested in the literatures for non-

K. Khandelwal · V. Mathur $(\bowtie)$

Department of Mathematics, Malaviya National Institute of Technology, Jaipur 302017, Rajasthan, India e-mail: vatsalamathurmnit@gmail.com

K. Khandelwal

e-mail: kavitakh21@gmail.com 
Newtonian fluids such as rate type [1], differential type [2] and integral type. These fluids have non-linear relationship between shear stress and the rate of strain.

For non-Newtonian fluids, the first exact solutions corresponding to motion of second grade fluids in cylindrical domains seem to be those of [3]. Similarly, [4] and [5] proposed first exact solutions for Maxwell fluids and Oldroyd-B fluids respectively. The first exact solution for motion of non-Newtonian fluids that applies a constant shear stress to the fluid are those of [6] and [7] for second-grade fluids. Exact solutions for Taylor-Couette flow of a fractional second grade fluid in an annulus due to a time-dependent couple have been obtained by [8]. [9] worked on unsteady rotating flows of a viscoelastic fluid with the fractional Maxwell model between coaxial cylinders. The velocity field and the associated tangential stress corresponding to the rotational flow of a generalized second grade fluid within an infinite circular cylinder have been obtained by [10]. Various other studies have been done recently on non-Newtonian fluids [11-18].

Fractional calculus approach is very important in describing viscoelasticity [19-21]. The starting point of the fractional derivative model of viscoelastic fluid is usually a classical differential equation. This is being modified by replacing the time derivative of an integer order by the so-called Riemann-Liouville fractional calculus operators. Hence, many exact solutions for non-Newtonian fluids with fractional derivatives have been established [22-29] due to the importance of viscoelasticity.

The aim of this paper is to provide exact solutions for the velocity field and shear stress corresponding to the unsteady flow of an incompressible fractional Maxwell fluid in annular region between two infinitely long coaxial circular cylinders. At time $t=0^{+}$, the inner cylinder applies a time dependent torsional shear to the fluid and outer cylinder is moving at a constant velocity. This solution is obtained using finite Hankel and Laplace transform methods and the result is presented in terms of the generalized-G and $\mathrm{R}$ functions. The solution of ordinary Maxwell fluids and Newtonian fluids are obtained by limiting cases of $\alpha \rightarrow 1$ and $\alpha \rightarrow 1, \lambda \rightarrow 0$ respectively.

\section{Governing Equations}

Let us consider an incompressible fractional Maxwell fluid with velocity $V$ and extra stress $\mathrm{S}$ as in the form of

$$
V=V(r, t)=w(r, t) e_{\theta}, \quad \mathrm{S}=\mathrm{S}(r, t),
$$

where $e_{\theta}$ is the unit vector in the $\theta$ direction of the cylindrical coordinates.

At time $t=0$, the fluid is at rest in an annular region between two infinite coaxial circular cylinders. At time $\mathrm{t}=0^{+}$, the inner cylinder applies a time dependent torsional shear to the fluid and outer cylinder is moving at a constant velocity. For these flows, the constraint of incompressibility is automatically satisfied. Initially the fluid is at rest, hence

$$
V(r, 0)=0, \quad \mathrm{~S}(r, 0)=0 .
$$

For such flows the constraint of incompressibility is automatically satisfied, while the governing equations [30] are

$$
\begin{aligned}
\left(1+\lambda D_{t}^{\alpha}\right) \frac{\partial w(r, t)}{\partial t} & =v\left(\frac{\partial^{2}}{\partial r^{2}}+\frac{1}{r} \frac{\partial}{\partial r}-\frac{1}{r^{2}}\right) w(r, t), \quad \mathrm{r} \in\left(\mathrm{R}_{1}, \mathrm{R}_{2}\right), \mathrm{t}>0 \\
\left(1+\lambda D_{t}^{\alpha}\right) \tau(r, t) & =\mu\left(\frac{\partial}{\partial r}-\frac{1}{r}\right) w(r, t), \quad \mathrm{r} \in\left(\mathrm{R}_{1}, \mathrm{R}_{2}\right), \mathrm{t}>0
\end{aligned}
$$


where $\tau(r, t)=S_{r \theta}(r, t)$ is the non-trivial shear stress, $\lambda$ is relaxation time, $\mu$ is the dynamic viscosity, $\rho$ is the constant density of the fluid, $v=\frac{\mu}{\rho}$ is the kinematic viscosity and $D_{t}^{\alpha}$ is the Caputo fractional derivative of order $\alpha$ as defined by [31]

$$
D_{t}^{\alpha} f(t)= \begin{cases}\frac{1}{\Gamma(1-\alpha)} \frac{d}{d t} \int_{0}^{t} \frac{f(\tau)}{(t-\tau)^{\alpha}} d \tau, & 0 \leq \alpha<1 \\ \frac{d}{d t} f(t), & \alpha=1,\end{cases}
$$

where $\Gamma($.$) is the Gamma function.$

For $\alpha \rightarrow 1$ when $D_{t}^{\alpha} f(t) \rightarrow d f(t) / d t$, Eqs. (3) and (4) are reduced to the governing equations for an Ordinary Maxwell fluid.

\section{Flow Through the Annular Region}

Let us consider an incompressible fractional Maxwell fluid at rest in the annular region between two infinite coaxial circular cylinders. Also, consider that radius of inner and outer cylinders are $R_{1}$ and $R_{2}\left(>R_{1}\right)$ respectively. At time $t=0^{+}$, the outer cylinder moving at a constant velocity and the inner cylinder begins to rotate about its axis with a time dependent torque per unit length $2 \pi R_{1} \tau\left(R_{1}, t\right)$ [30], where

$$
\tau\left(R_{1}, t\right)=\frac{f_{1}}{\lambda} R_{\alpha,-1}\left(-\frac{1}{\lambda}, t\right) ; \quad 0<\alpha<1,
$$

where $f_{1}$ is a constant and generalized $\mathrm{R}$ functions are defined by [32]

$$
\begin{aligned}
& R_{a, b}(d, t)=L^{-1}\left\{\frac{q^{b}}{q^{a}-d}\right\}=\sum_{n=0}^{\infty} \frac{d^{n} t^{(n+1) a-b-1}}{\Gamma[(n+1) a-b]} \\
& \operatorname{Re}(a-b)>0, \operatorname{Re}(\mathrm{q})>0,\left|\frac{\mathrm{d}}{\mathrm{q}^{\mathrm{a}}}\right|<1 .
\end{aligned}
$$

The governing equations are given by Eqs. (3) and (4), while appropriate initial and boundary conditions are

$$
w(r, 0)=\frac{\partial w(r, 0)}{\partial t}=0, \tau(\mathrm{r}, 0)=0, \mathrm{r} \in\left(\mathrm{R}_{1}, \mathrm{R}_{2}\right],
$$

and

$$
\begin{gathered}
\left.\left(1+\lambda D_{t}^{\alpha}\right) \tau(r, t)\right|_{r=R_{1}}=\left.\mu\left(\frac{\partial}{\partial r}-\frac{1}{r}\right) w(r, t)\right|_{r=R_{1}}=f_{1}, \\
w\left(R_{2}, t\right)=f_{2}, \mathrm{t}>0,
\end{gathered}
$$

where $f_{2}$ is the constant velocity of outer cylinder. Eq. (6) is the solution of Eq. (9). To solve this problem we use Laplace and Hankel transform methods.

\section{Calculation of the Velocity Field}

Applying Laplace transform of Eq. (3) and using the initial conditions as given in Eq. (8), we obtain

$$
\left(q+\lambda q^{\alpha+1}\right) \bar{w}(r, q)=v\left(\frac{\partial^{2}}{\partial r^{2}}+\frac{1}{r} \frac{\partial}{\partial r}-\frac{1}{r^{2}}\right) \bar{w}(r, q), \mathrm{r} \in\left(\mathrm{R}_{1}, \mathrm{R}_{2}\right) .
$$


where $\bar{w}(r, q)=\int_{0}^{\infty} e^{-q t} w(r, t) d t$ is the Laplace transform of function $w(r, t)$ and $q$ is the transform parameter.

Applying Laplace transform of Eq. (9), we obtain

$$
\begin{gathered}
\left.\left(\frac{\partial}{\partial r}-\frac{1}{r}\right) \bar{w}(r, q)\right|_{r=R_{1}}=\frac{f_{1}}{\mu q} ; \\
\bar{w}\left(R_{2}, q\right)=\frac{f_{2}}{q} .
\end{gathered}
$$

The Hankel transform method with respect to $r$ is used and defined as follows

$$
\bar{w}_{H}\left(r_{n}, q\right)=\int_{R_{1}}^{R_{2}} r \bar{w}(r, q) B\left(r, r_{n}\right) d r
$$

where

$$
B\left(r, r_{n}\right)=J_{1}\left(r r_{n}\right) Y_{2}\left(R_{1} r_{n}\right)-J_{2}\left(R_{1} r_{n}\right) Y_{1}\left(r r_{n}\right),
$$

$r_{n}$ being the positive roots of the transcendental equation $B\left(R_{2}, r\right)=0$. The inverse Hankel transform as defined by [30], is given below

$$
\bar{w}(r, q)=\frac{\pi^{2}}{2} \sum_{n=1}^{\infty} \frac{r_{n}^{2} J_{1}^{2}\left(R_{2} r_{n}\right) B\left(r, r_{n}\right)}{J_{2}^{2}\left(R_{1} r_{n}\right)-J_{1}^{2}\left(R_{2} r_{n}\right)} \bar{w}_{H}\left(r_{n}, q\right) .
$$

Multiplying both sides of Eq. (10) by $r B\left(r, r_{n}\right)$, then integrating with respect to $r$ from $R_{1}$ to $R_{2}$ and taking into account the conditions Eq. (11) and the equality

$$
\begin{aligned}
\int_{R_{1}}^{R_{2}} r & \left(\frac{\partial^{2}}{\partial r^{2}}+\frac{1}{r} \frac{\partial}{\partial r}-\frac{1}{r^{2}}\right) \bar{w}(r, q) B\left(r, r_{n}\right) d r \\
= & -r_{n}^{2} \bar{w}_{H}\left(r_{n}, q\right)+\left.\frac{2}{\pi r_{n}}\left(\frac{\partial}{\partial r}-\frac{1}{r}\right) \bar{w}(r, q)\right|_{r=R_{1}}+R_{2} r_{n} \bar{w}\left(R_{2}, q\right)\left[Y_{2}\left(R_{1} r_{n}\right) J_{2}\left(R_{2} r_{n}\right)\right. \\
& \left.-J_{2}\left(R_{1} r_{n}\right) Y_{2}\left(R_{2} r_{n}\right)\right] \\
= & -r_{n}^{2} \bar{w}_{H}\left(r_{n}, q\right)+\frac{2}{\pi r_{n}} \frac{f_{1}}{\mu q}+\frac{R_{2} r_{n} f_{2}}{q}\left[Y_{2}\left(R_{1} r_{n}\right) J_{2}\left(R_{2} r_{n}\right)-J_{2}\left(R_{1} r_{n}\right) Y_{2}\left(R_{2} r_{n}\right)\right], \quad(15)
\end{aligned}
$$

we obtain

$$
\begin{aligned}
& \bar{w}_{H}\left(r_{n}, q\right) \\
& =\frac{2 f_{1} v}{\pi \mu r_{n}} \frac{1}{q\left(q+\lambda q^{\alpha+1}+v r_{n}^{2}\right)} \\
& \quad+v R_{2} r_{n} f_{2}\left[Y_{2}\left(R_{1} r_{n}\right) J_{2}\left(R_{2} r_{n}\right)-J_{2}\left(R_{1} r_{n}\right) Y_{2}\left(R_{2} r_{n}\right)\right] \frac{1}{q\left(q+\lambda q^{\alpha+1}+v r_{n}^{2}\right)} .
\end{aligned}
$$


Rewriting Eq. (16) into a suitable equivalent form, we obtain below

$$
\begin{aligned}
& \bar{w}_{H}\left(r_{n}, q\right) \\
& =\frac{2 f_{1}}{\pi \mu r_{n}^{3}} \frac{1}{q}-\frac{2 f_{1}\left(1+\lambda q^{\alpha}\right)}{\pi \mu r_{n}^{3}\left(q+\lambda q^{\alpha+1}+v r_{n}^{2}\right)} \\
& \quad+\frac{f_{2} R_{2}}{r_{n}}\left[Y_{2}\left(R_{1} r_{n}\right) J_{2}\left(R_{2} r_{n}\right)-J_{2}\left(R_{1} r_{n}\right) Y_{2}\left(R_{2} r_{n}\right)\right]\left[\frac{1}{q}-\frac{\left(1+\lambda q^{\alpha}\right)}{\left(q+\lambda q^{\alpha+1}+v r_{n}^{2}\right)}\right] .
\end{aligned}
$$

Applying inverse Hankel transform to Eq. (17) and taking into account the following result

$$
\int_{R_{1}}^{R_{2}}\left(r^{2}-R_{2}^{2}\right) B\left(r, r_{n}\right) d r=\frac{4}{\pi r_{n}^{3}}\left(\frac{R_{2}}{R_{1}}\right)^{2},
$$

we obtain

$$
\begin{aligned}
\bar{w}(r, q) & \frac{f_{1}}{2 \mu}\left(\frac{R_{1}}{R_{2}}\right)^{2}\left(r-\frac{R_{2}^{2}}{r}\right) \frac{1}{q}-\frac{\pi f_{1}}{\mu} \sum_{n=1}^{\infty} \frac{J_{1}^{2}\left(R_{2} r_{n}\right) B\left(r, r_{n}\right)}{r_{n}\left[J_{2}^{2}\left(R_{1} r_{n}\right)-J_{1}^{2}\left(R_{2} r_{n}\right)\right]} \frac{\left(1+\lambda q^{\alpha}\right)}{\left(q+\lambda q^{\alpha+1}+v r_{n}^{2}\right)} \\
& +\frac{\pi^{2}}{2} R_{2} f_{2} \sum_{n=1}^{\infty} \frac{r_{n} J_{1}^{2}\left(R_{2} r_{n}\right) B\left(r, r_{n}\right)}{\left[J_{2}^{2}\left(R_{1} r_{n}\right)-J_{1}^{2}\left(R_{2} r_{n}\right)\right]}\left[Y_{2}\left(R_{1} r_{n}\right) J_{2}\left(R_{2} r_{n}\right)-J_{2}\left(R_{1} r_{n}\right) Y_{2}\left(R_{2} r_{n}\right)\right] \\
& \times\left[\frac{1}{q}-\frac{\left(1+\lambda q^{\alpha}\right)}{\left(q+\lambda q^{\alpha+1}+v r_{n}^{2}\right)}\right] .
\end{aligned}
$$

Applying Inverse-Laplace transform of Eq. (19) and taking into account the following result [32]

$$
\begin{aligned}
G_{a, b, c}(d, t)= & L^{-1}\left\{\frac{q^{b}}{\left(q^{a}-d\right)^{c}}\right\} \\
= & \sum_{\mathrm{j}=0}^{\infty} \frac{\mathrm{d}^{\mathrm{j}} \Gamma(c+j)}{\Gamma(c) \Gamma(j+1)} \frac{t^{(c+j) a-b-1}}{\Gamma[(c+j) a-b]} ; \\
& \operatorname{Re}(a c-b)>0, \operatorname{Re}(\mathrm{q})>0,\left|\frac{\mathrm{d}}{\mathrm{q}^{\mathrm{a}}}\right|<1,
\end{aligned}
$$

we obtain

$$
\begin{aligned}
w(r, t) & \\
= & \frac{f_{1}}{2 \mu}\left(\frac{R_{1}}{R_{2}}\right)^{2}\left(r-\frac{R_{2}^{2}}{r}\right)-\frac{\pi f_{1}}{\mu \lambda} \sum_{n=1}^{\infty} \frac{J_{1}^{2}\left(R_{2} r_{n}\right) B\left(r, r_{n}\right)}{r_{n}\left[J_{2}^{2}\left(R_{1} r_{n}\right)-J_{1}^{2}\left(R_{2} r_{n}\right)\right]} \sum_{k=0}^{\infty}\left(\frac{-v r_{n}^{2}}{\lambda}\right)^{k} \\
& \times\left[G_{\alpha,-k-1, k+1}\left(-\lambda^{-1}, t\right)+\lambda G_{\alpha, \alpha-k-1, k+1}\left(-\lambda^{-1}, t\right)\right] \\
& +\frac{\pi^{2}}{2} R_{2} f_{2} \sum_{n=1}^{\infty} \frac{r_{n} J_{1}^{2}\left(R_{2} r_{n}\right) B\left(r, r_{n}\right)}{\left[J_{2}^{2}\left(R_{1} r_{n}\right)-J_{1}^{2}\left(R_{2} r_{n}\right)\right]}\left[Y_{2}\left(R_{1} r_{n}\right) J_{2}\left(R_{2} r_{n}\right)-J_{2}\left(R_{1} r_{n}\right) Y_{2}\left(R_{2} r_{n}\right)\right] \\
& \times\left[1-\frac{1}{\lambda} \sum_{k=0}^{\infty}\left(\frac{-v r_{n}^{2}}{\lambda}\right)^{k}\left\{G_{\alpha,-k-1, k+1}\left(-\lambda^{-1}, t\right)+\lambda G_{\alpha, \alpha-k-1, k+1}\left(-\lambda^{-1}, t\right)\right\}\right] .
\end{aligned}
$$



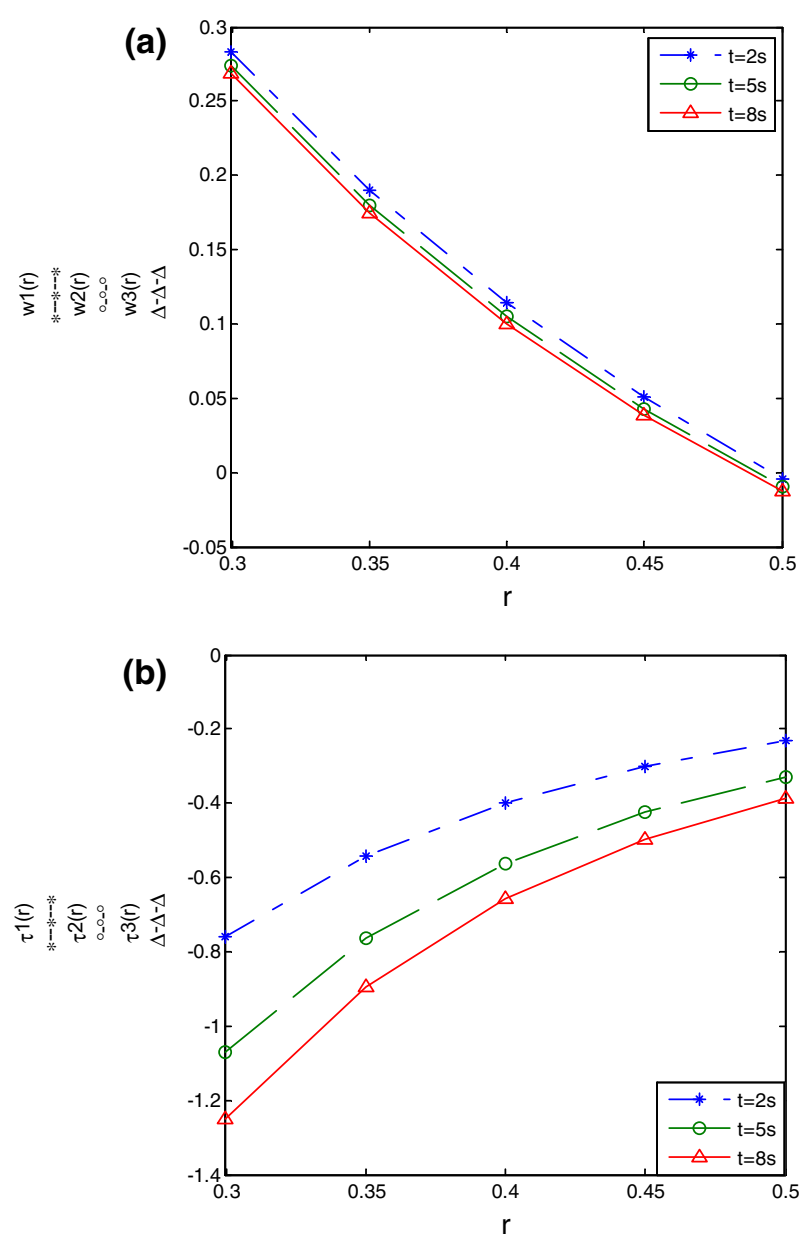

Fig. 1 Profiles of the velocity $\mathrm{w}(\mathrm{r}, \mathrm{t})$ and shear stress $\tau(\mathrm{r}, \mathrm{t})$ given by Eqs. (21) and (25) for $R_{1}=0.3, R_{2}=$ $0.5, f_{1}=-3, f_{2}=-2, v=0.015, \mu=1.01, \lambda=5, \alpha=0.5$ and different values of $\mathrm{t}$

Calculation of the shear stress

Applying Laplace transform to Eq. (4), we obtain

$$
\bar{\tau}(r, q)=\mu \frac{1}{\left(1+\lambda q^{\alpha}\right)}\left(\frac{\partial}{\partial r}-\frac{1}{r}\right) \bar{w}(r, q) .
$$

Substitute Eq. (19) in Eq. (22), we obtain

$$
\begin{aligned}
\bar{\tau}(r, q)= & f_{1}\left(\frac{R_{1}}{r}\right)^{2} \frac{1}{q\left(1+\lambda q^{\alpha}\right)}+\pi f_{1} \sum_{n=1}^{\infty} \frac{J_{1}^{2}\left(R_{2} r_{n}\right) \bar{B}\left(r, r_{n}\right)}{\left[J_{2}^{2}\left(R_{1} r_{n}\right)-J_{1}^{2}\left(R_{2} r_{n}\right)\right]} \frac{1}{\left(q+\lambda q^{\alpha+1}+v r_{n}^{2}\right)} \\
& -\frac{\pi^{2}}{2} \mu R_{2} f_{2} \sum_{n=1}^{\infty} \frac{r_{n}^{2} J_{1}^{2}\left(R_{2} r_{n}\right) \bar{B}\left(r, r_{n}\right)}{\left[J_{2}^{2}\left(R_{1} r_{n}\right)-J_{1}^{2}\left(R_{2} r_{n}\right)\right]}\left[Y_{2}\left(R_{1} r_{n}\right) J_{2}\left(R_{2} r_{n}\right)\right.
\end{aligned}
$$



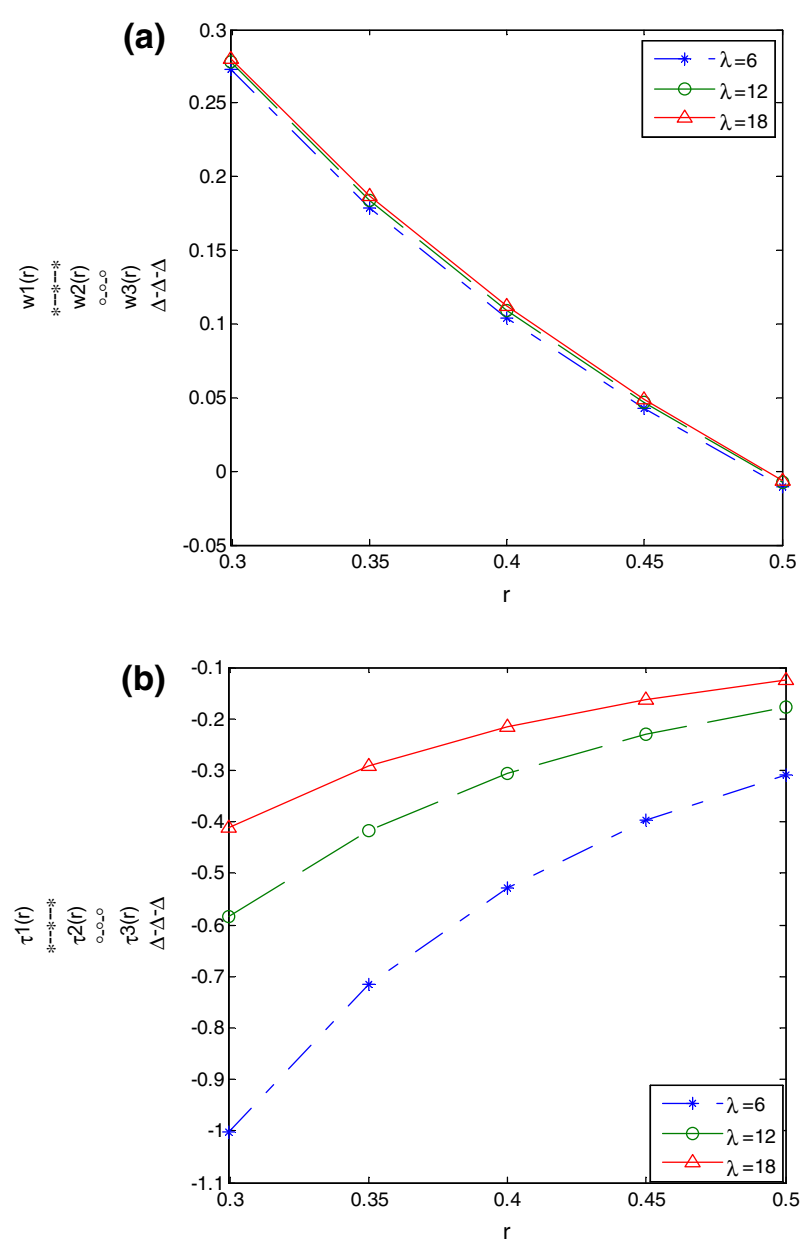

Fig. 2 Profiles of the velocity $\mathrm{w}(\mathrm{r}, \mathrm{t})$ and shear stress $\tau(\mathrm{r}, \mathrm{t})$ given by Eqs. (21) and (25) for $R_{1}=0.3, R_{2}=$ $0.5, f_{1}=-3, f_{2}=-2, \mathrm{t}=6 \mathrm{~s}, v=0.015, \mu=1.01, \alpha=0.5$ and different values of $\lambda$

$$
\begin{aligned}
& \left.-J_{2}\left(R_{1} r_{n}\right) Y_{2}\left(R_{2} r_{n}\right)\right] \\
& \times\left[\frac{1}{q\left(1+\lambda q^{\alpha}\right)}-\frac{1}{\left(q+\lambda q^{\alpha+1}+v r_{n}^{2}\right)}\right],
\end{aligned}
$$

where

$$
\bar{B}\left(r, r_{n}\right)=J_{2}\left(r r_{n}\right) Y_{2}\left(R_{1} r_{n}\right)-J_{2}\left(R_{1} r_{n}\right) Y_{2}\left(r r_{n}\right)
$$

Applying inverse Laplace transform to Eq. (23) and using Eq. (20), we obtain

$$
\begin{aligned}
\tau(r, t)= & f_{1}\left(\frac{R_{1}}{r}\right)^{2} \frac{1}{\lambda} R_{\alpha,-1}\left(-\lambda^{-1}, t\right) \\
& +\frac{\pi f_{1}}{\lambda} \sum_{n=1}^{\infty} \frac{J_{1}^{2}\left(R_{2} r_{n}\right) \bar{B}\left(r, r_{n}\right)}{\left[J_{2}^{2}\left(R_{1} r_{n}\right)-J_{1}^{2}\left(R_{2} r_{n}\right)\right]} \sum_{k=0}^{\infty}\left(\frac{-v r_{n}^{2}}{\lambda}\right)^{k} G_{\alpha,-k-1, k+1}\left(-\lambda^{-1}, t\right)
\end{aligned}
$$



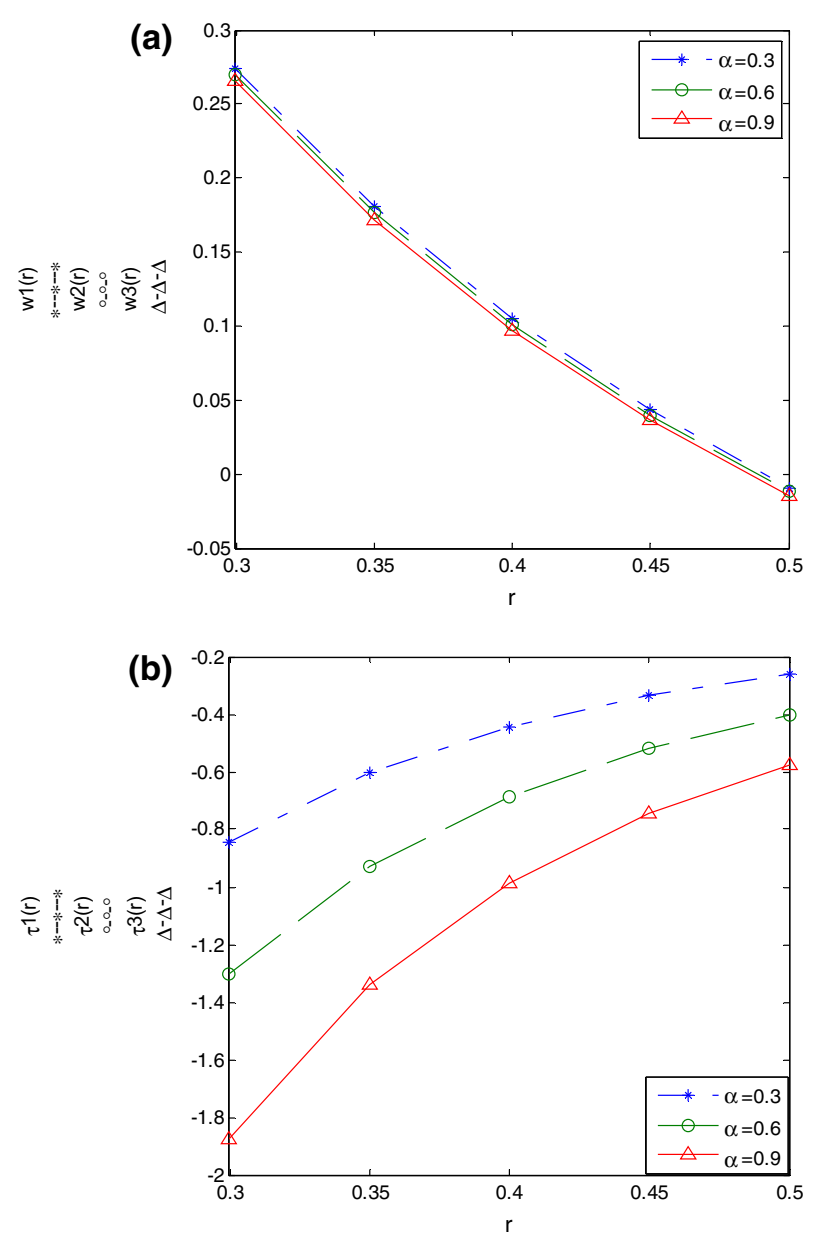

Fig. 3 Profiles of the velocity $w(r, \mathrm{t})$ and shear stress $\tau(\mathrm{r}, \mathrm{t})$ given by Eqs. (21) and (25) for $R_{1}=0.3, R_{2}=$ $0.5, f_{1}=-3, f_{2}=-2, \mathrm{t}=6 \mathrm{~s}, v=0.015, \mu=1.01, \lambda=5$ and different values of $\alpha$

$$
\begin{aligned}
- & \frac{\pi^{2}}{2} \frac{\mu R_{2} f_{2}}{\lambda} \sum_{n=1}^{\infty} \frac{r_{n}^{2} J_{1}^{2}\left(R_{2} r_{n}\right) \bar{B}\left(r, r_{n}\right)}{\left[J_{2}^{2}\left(R_{1} r_{n}\right)-J_{1}^{2}\left(R_{2} r_{n}\right)\right]} \\
& {\left[Y_{2}\left(R_{1} r_{n}\right) J_{2}\left(R_{2} r_{n}\right)-J_{2}\left(R_{1} r_{n}\right) Y_{2}\left(R_{2} r_{n}\right)\right] } \\
\times & {\left[R_{\alpha,-1}\left(-\lambda^{-1}, t\right)-\sum_{k=0}^{\infty}\left(\frac{-v r_{n}^{2}}{\lambda}\right)^{k} G_{\alpha,-k-1, k+1}\left(-\lambda^{-1}, t\right)\right] . }
\end{aligned}
$$

\section{Limiting Cases}

Ordinary Maxwell Fluid

Applying $\alpha \rightarrow 1$ into Eqs. (21) and (25), we obtain the velocity field 

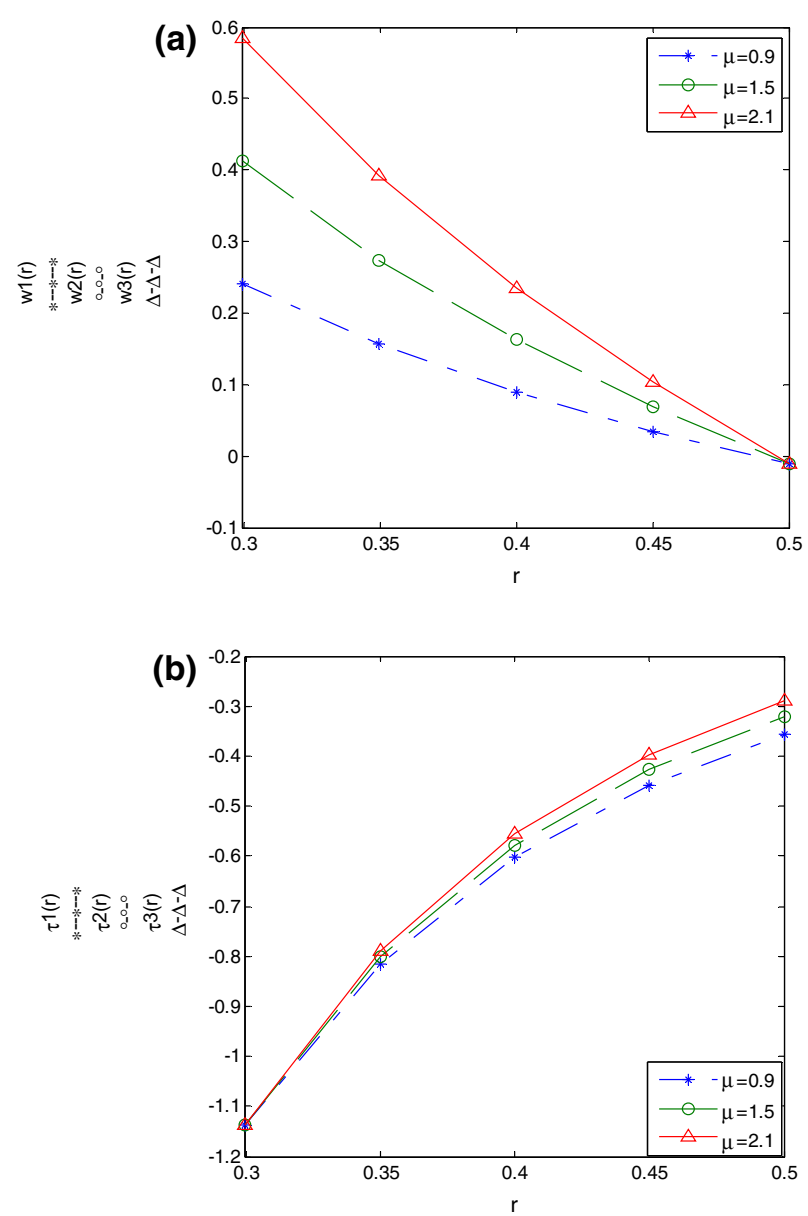

Fig. 4 Profiles of the velocity $\mathrm{w}(\mathrm{r}, \mathrm{t})$ and shear stress $\tau(\mathrm{r}, \mathrm{t})$ given by Eqs. (21) and (25) for $R_{1}=0.3, R_{2}=$ $0.5, f_{1}=-3, f_{2}=-2, \mathrm{t}=6 \mathrm{~s}, v=0.015, \lambda=5, \alpha=0.5$ and different values of $\mu$

$$
\begin{aligned}
& w_{M}(r, t) \\
& =\frac{f_{1}}{2 \mu}\left(\frac{R_{1}}{R_{2}}\right)^{2}\left(r-\frac{R_{2}^{2}}{r}\right)-\frac{\pi f_{1}}{\mu \lambda} \sum_{n=1}^{\infty} \frac{J_{1}^{2}\left(R_{2} r_{n}\right) B\left(r, r_{n}\right)}{r_{n}\left[J_{2}^{2}\left(R_{1} r_{n}\right)-J_{1}^{2}\left(R_{2} r_{n}\right)\right]} \sum_{k=0}^{\infty}\left(\frac{-v r_{n}^{2}}{\lambda}\right)^{k} \\
& \quad \times\left[G_{1,-k-1, k+1}\left(-\lambda^{-1}, t\right)+\lambda G_{1,-k, k+1}\left(-\lambda^{-1}, t\right)\right] \\
& \quad+\frac{\pi^{2}}{2} R_{2} f_{2} \sum_{n=1}^{\infty} \frac{r_{n} J_{1}^{2}\left(R_{2} r_{n}\right) B\left(r, r_{n}\right)}{\left[J_{2}^{2}\left(R_{1} r_{n}\right)-J_{1}^{2}\left(R_{2} r_{n}\right)\right]}\left[Y_{2}\left(R_{1} r_{n}\right) J_{2}\left(R_{2} r_{n}\right)-J_{2}\left(R_{1} r_{n}\right) Y_{2}\left(R_{2} r_{n}\right)\right] \\
& \quad \times\left[1-\frac{1}{\lambda} \sum_{k=0}^{\infty}\left(\frac{-v r_{n}^{2}}{\lambda}\right)^{k}\left\{G_{1,-k-1, k+1}\left(-\lambda^{-1}, t\right)+\lambda G_{1,-k, k+1}\left(-\lambda^{-1}, t\right)\right\}\right],
\end{aligned}
$$

and its associated shear stress corresponding to ordinary Maxwell fluid performing the same motion 

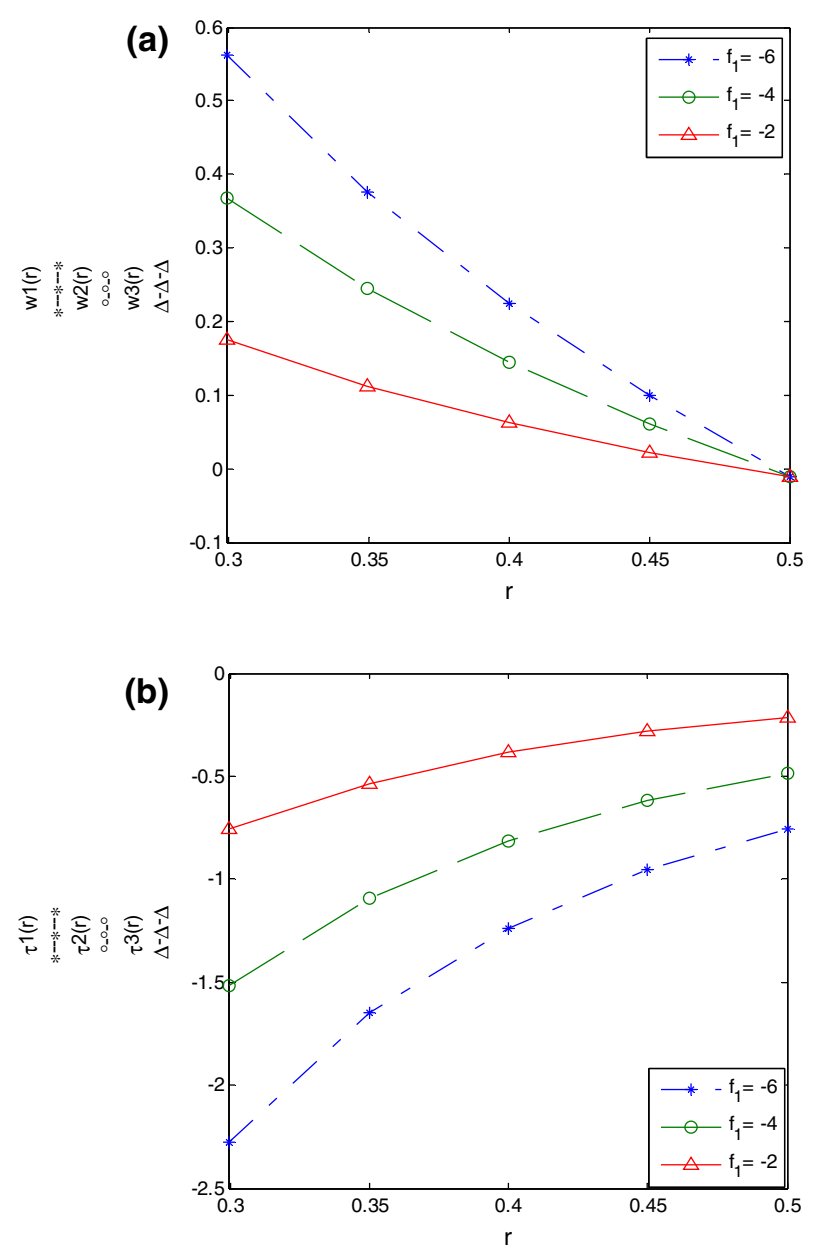

Fig. 5 Profiles of the velocity $\mathrm{w}(\mathrm{r}, \mathrm{t})$ and shear stress $\tau(\mathrm{r}, \mathrm{t})$ given by Eqs. (21) and (25) for $R_{1}=0.3, R_{2}=$ $0.5, f_{2}=-2, \mathrm{t}=6 \mathrm{~s}, v=0.015, \mu=1.01, \lambda=5, \alpha=0.5$ and different values of $f_{1}$

$$
\begin{aligned}
\tau_{M}(r, t)= & f_{1}\left(\frac{R_{1}}{r}\right)^{2}\left(1-e^{-t / \lambda}\right) \\
& +\frac{\pi f_{1}}{\lambda} \sum_{n=1}^{\infty} \frac{J_{1}^{2}\left(R_{2} r_{n}\right) \bar{B}\left(r, r_{n}\right)}{\left[J_{2}^{2}\left(R_{1} r_{n}\right)-J_{1}^{2}\left(R_{2} r_{n}\right)\right]} \sum_{k=0}^{\infty}\left(\frac{-v r_{n}^{2}}{\lambda}\right)^{k} G_{1,-k-1, k+1}\left(-\lambda^{-1}, t\right) \\
& -\frac{\pi^{2}}{2} \frac{\mu R_{2} f_{2}}{\lambda} \sum_{n=1}^{\infty} \frac{r_{n}^{2} J_{1}^{2}\left(R_{2} r_{n}\right) \bar{B}\left(r, r_{n}\right)}{\left[J_{2}^{2}\left(R_{1} r_{n}\right)-J_{1}^{2}\left(R_{2} r_{n}\right)\right]}\left[Y_{2}\left(R_{1} r_{n}\right) J_{2}\left(R_{2} r_{n}\right)\right. \\
& \left.-J_{2}\left(R_{1} r_{n}\right) Y_{2}\left(R_{2} r_{n}\right)\right] \\
& \times\left[\lambda\left(1-e^{-t / \lambda}\right)-\sum_{k=0}^{\infty}\left(\frac{-v r_{n}^{2}}{\lambda}\right)^{k} G_{1,-k-1, k+1}\left(-\lambda^{-1}, t\right)\right] .
\end{aligned}
$$



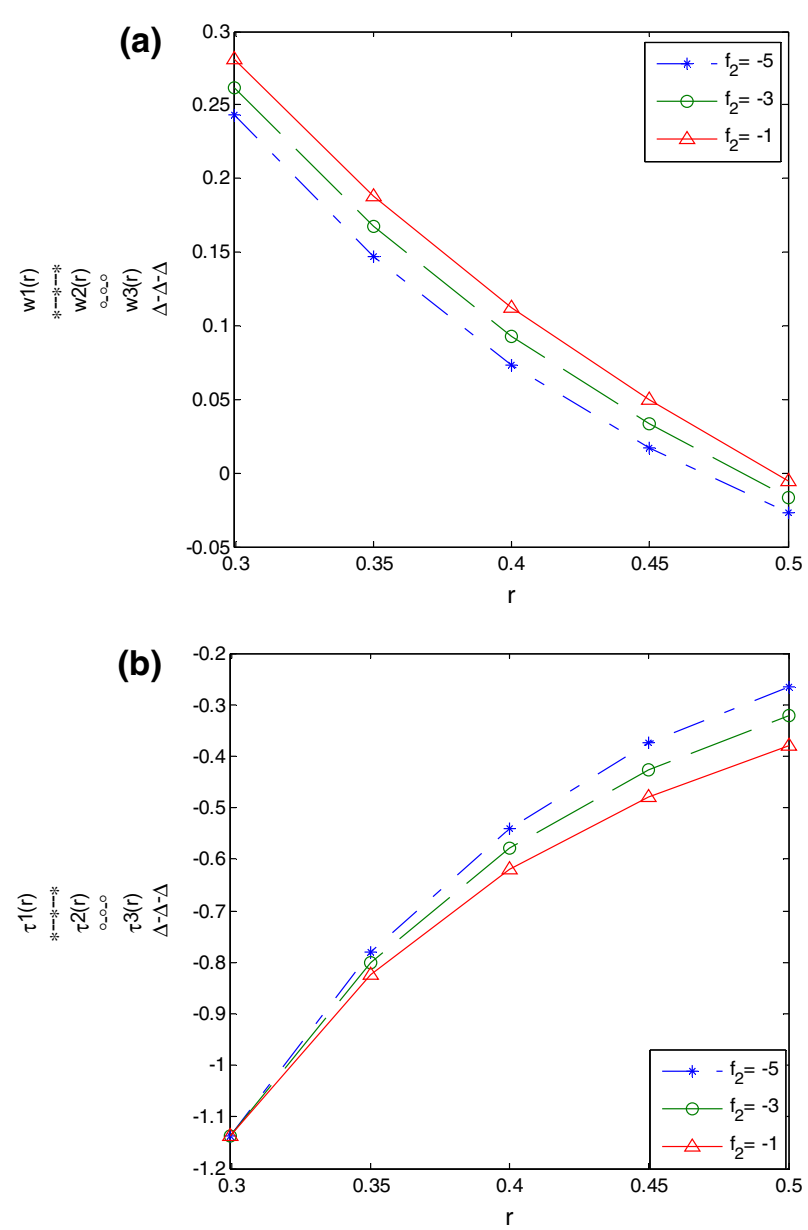

Fig. 6 Profiles of the velocity $\mathrm{w}(\mathrm{r}, \mathrm{t})$ and shear stress $\tau(\mathrm{r}, \mathrm{t})$ given by Eqs. (21) and (25) for $R_{1}=0.3, R_{2}=$ $0.5, f_{1}=-3, \mathrm{t}=6 \mathrm{~s}, v=0.015, \mu=1.01, \lambda=5, \alpha=0.5$ and different values of $f_{2}$

\section{Newtonian Fluid}

Applying $\lambda \rightarrow 0$ into Eqs. (26) and (27) and taking into account the following result

$$
\lim _{\lambda \rightarrow 0} \frac{1}{\lambda^{m}} G_{1, b, m}\left(-\lambda^{-1}, t\right)=\frac{t^{-b-1}}{\Gamma(-b)}, \mathrm{b}<0,
$$

we obtain the corresponding solutions for the Newtonian fluid, as follows

$$
\begin{aligned}
w_{N}(r, t)= & \frac{f_{1}}{2 \mu}\left(\frac{R_{1}}{R_{2}}\right)^{2}\left(r-\frac{R_{2}^{2}}{r}\right)-\frac{\pi f_{1}}{\mu} \sum_{n=1}^{\infty} \frac{J_{1}^{2}\left(R_{2} r_{n}\right) B\left(r, r_{n}\right)}{r_{n}\left[J_{2}^{2}\left(R_{1} r_{n}\right)-J_{1}^{2}\left(R_{2} r_{n}\right)\right]} e^{-v r_{n}^{2} t} \\
& +\frac{\pi^{2}}{2} R_{2} f_{2} \sum_{n=1}^{\infty} \frac{r_{n} J_{1}^{2}\left(R_{2} r_{n}\right) B\left(r, r_{n}\right)}{\left[J_{2}^{2}\left(R_{1} r_{n}\right)-J_{1}^{2}\left(R_{2} r_{n}\right)\right]}\left[Y_{2}\left(R_{1} r_{n}\right) J_{2}\left(R_{2} r_{n}\right)\right. \\
& \left.-J_{2}\left(R_{1} r_{n}\right) Y_{2}\left(R_{2} r_{n}\right)\right]\left(1-e^{-v r_{n}^{2} t}\right),
\end{aligned}
$$



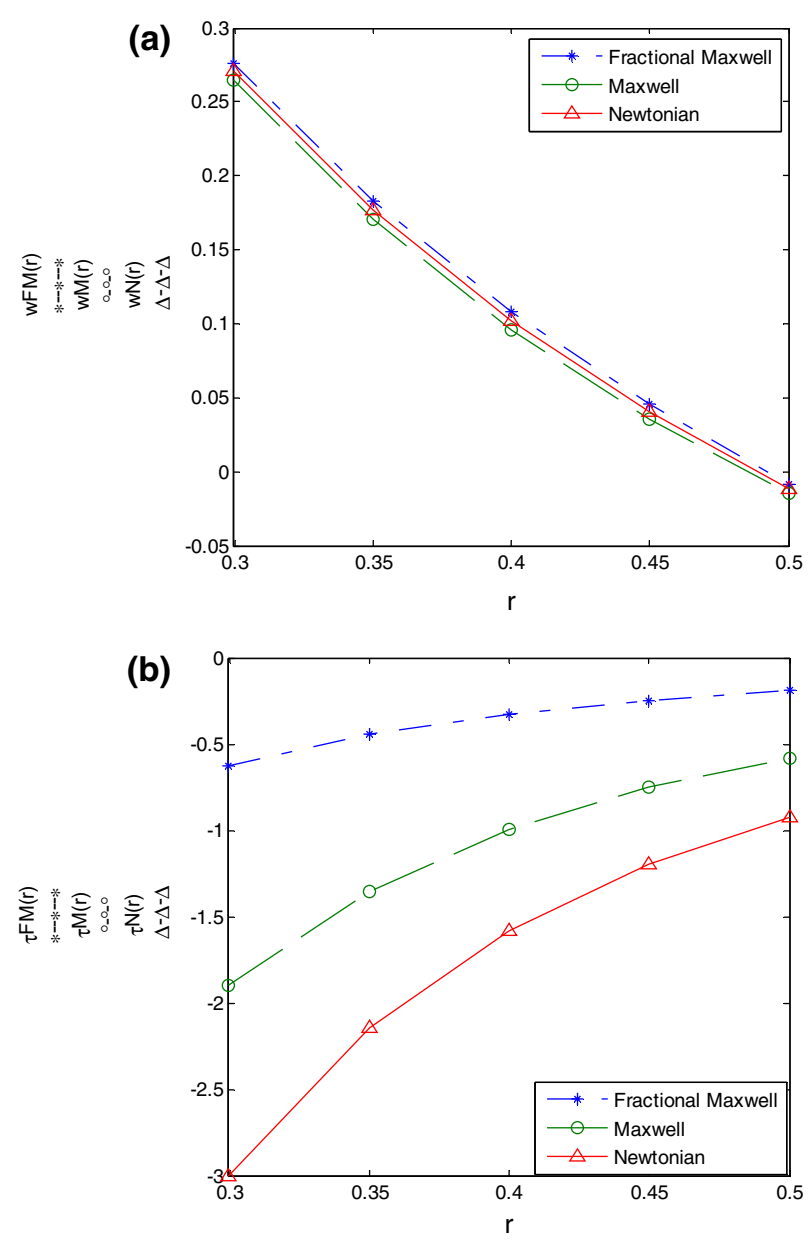

Fig. 7 Profiles of the velocity $w(r, t)$ and shear stress $\tau(r, t)$ corresponding to the Fractional Maxwell, ordinary Maxwell and Newtonian fluids, for $R_{1}=0.3, R_{2}=0.5, f_{1}=-3, f_{2}=-2, \mathrm{t}=6 \mathrm{~s}, v=0.015, \mu=$ $1.01, \lambda=6$ and $\alpha=0.2$

and

$$
\begin{aligned}
\tau_{N}(r, t)= & f_{1}\left(\frac{R_{1}}{r}\right)^{2}+\pi f_{1} \sum_{n=1}^{\infty} \frac{J_{1}^{2}\left(R_{2} r_{n}\right) \bar{B}\left(r, r_{n}\right)}{\left[J_{2}^{2}\left(R_{1} r_{n}\right)-J_{1}^{2}\left(R_{2} r_{n}\right)\right]} e^{-v r_{n}^{2} t} \\
& -\frac{\pi^{2}}{2} \mu R_{2} f_{2} \sum_{n=1}^{\infty} \frac{r_{n}^{2} J_{1}^{2}\left(R_{2} r_{n}\right) \bar{B}\left(r, r_{n}\right)}{\left[J_{2}^{2}\left(R_{1} r_{n}\right)-J_{1}^{2}\left(R_{2} r_{n}\right)\right]}\left[Y_{2}\left(R_{1} r_{n}\right) J_{2}\left(R_{2} r_{n}\right)\right. \\
& \left.-J_{2}\left(R_{1} r_{n}\right) Y_{2}\left(R_{2} r_{n}\right)\right]\left(1-e^{-v r_{n}^{2} t}\right) .
\end{aligned}
$$

\section{Conclusions and Numerical Results}

The purpose of this paper is to establish exact solutions for the velocity field and shear stress corresponding to the unsteady flow of an incompressible fractional Maxwell fluid flow 
in the annular region. Where, the motion is produced by the inner cylinder that applies a time dependent torsional shear to the fluid and outer cylinder which is moving at a constant velocity. The solution is obtained by finite Hankel and Laplace transform methods and the result is presented under series form in terms of the generalized $\mathrm{G}$ and $\mathrm{R}$ functions. The similar solutions for Ordinary Maxwell and Newtonian fluids are also obtained as limiting cases of the solution for fractional Maxwell fluid. The velocity field and shear stress are also analyzed using graphical illustration for various parameters, constants and fractional coefficients and a comparison between models of the velocity field and shear stress are also analyzed using graphical illustration.

As shown in below diagrams, the velocity $w(r, t)$ and the shear stress $\tau(r, t)$ given by Eqs. (21) and (25) have been drawn against $r$ for different values of the time $t, f_{1}, f_{2}$ and other relevant parameters. It can be clearly seen from the figures that the velocity component $w$ is decreasing function of $r$ and the shear stress component $\tau$ is increasing function of $r$. The motion of the fluid is relatively higher and shear stress lower in the neighborhood of the inner cylinder for given boundary conditions and $f_{1}<0, f_{2}<0$. Figures $1 \mathrm{a}$ and $\mathrm{b}$ are showing the effect of different values of time on the fluid motion. It can be seen that the velocity and the shear stress are the decreasing function of time $t$. The influence of relaxation time $\lambda$ and fractional parameter $\alpha$ on the fluid motion is shown in Figs. 2 and 3. Both parameters have opposite effect on the fluid motion. The velocity and the shear stress are increasing function of $\lambda$ and decreasing function of $\alpha$. Figures $4 a$ and $b$ are showing the effect of different values of dynamic viscosity on the fluid motion. The results indicate that the velocity and the shear stress are increasing function of dynamic viscosity. Figures 5 and 6 are showing the behavior of $f_{1}$ and $f_{2}$ on the fluid motion for their different values. Figure 7 is showing a comparison diagram of the velocity $w(r, t)$ and the shear stress $\tau(r, t)$ among three models (Fractional Maxwell fluid, Ordinary Maxwell fluid and Newtonian fluid) for same values of the common material constants and time t. The velocity in the neighborhood of inner cylinders is swiftest for fractional Maxwell fluid while it is slowest for the Ordinary Maxwell fluid. Similarly, shear stress on the whole flow domains highest for fractional Maxwell fluid while it is slowest for the Newtonian fluid.

In all of above, the root $r_{n}$ has been approximated by $\frac{(2 n-1) \pi}{2\left(R_{2}-R_{1}\right)}$.

\section{References}

1. Rajagopal, K.R., Srinivasa, A.R.: A thermodynamical frame-work for rate type fluid models. J. NonNewtonian Fluid Mech. 88, 207-227 (2000)

2. Dunn, J.E., Rajagopal, K.R.: Fluids of differential type: critical review and thermodynamic analysis. Int. J. Eng. Sci. 33, 689-729 (1995)

3. Ting, T.W.: Certain non-steady flows of second-order fluids. Arch. Ration. Mech. Anal. 14, 1-23 (1963)

4. Srivastava, P.N.: Non-steady Helical flow of a viscoelastic liquid. Arch. Mech. Stos. 18, 145-150 (1966)

5. Waters, N.D., King, M.J.: The unsteady flow of an Elastico-viscous liquid in a straight pipe of circular cross section. J. Phys. D Appl. Phys. 4, 204-211 (1971)

6. Bandelli, R., Rajagopal, K.R.: Start-up flows of second grade fluids in domains with one finite Dimension. Int. J. Non-Linear Mech. 30, 817-839 (1995)

7. Bandelli, R., Rajagopal, K.R., Galdi, G.P.: On some unsteady motions of fluids of second grade. Arch. Mech. 47, 661-676 (1995)

8. Imran, M., Kamran, M., Athar, M., Zafar, A.A.: Taylor-Couette flow of a fractional second grade fluid in an annulus due to a time-dependent couple. Nonlinear Anal. 16, 47-58 (2011)

9. Qi, H., Jin, H.: Unsteady rotating flows of a viscoelastic fluid with the fractional Maxwell model between coaxial cylinders. Acta Mechanica Sinica 22, 301-305 (2006)

10. Kamran, M., Imran, M., Athar, M.: Exact solutions for the unsteady rotational flow of a generalized second grade fluid through a circular cylinder. Nonlinear Anal. 15, 437-444 (2010) 
11. Amir, M., Fetecau, C., Imran, S.: Exact solutions for some unsteady flows of generalized second grade fluids in cylindrical domains. J. Prime Res. Math. 4, 171-180 (2008)

12. Kamran, M., Imran, M., Athar, M., Imran, M.A.: On the unsteady rotational flow of fractional Oldroyd-B fluid in cylindrical domains. Meccanica 47, 573-584 (2012)

13. Athar, M., Awan, A.U., Fetecau, C.: A note on the unsteady flow of a fractional Maxwell fluid through a circular cylinder. Acta Mech. Sin. 28, 308-314 (2012)

14. Athar, M., Kamran, M., Fetecau, C.: Taylor-Couette flow of a generalized second grade fluid due to a constant couple. Nonlinear Anal. 15, 3-13 (2010)

15. Athar, M., Fetecau, C., Kamran, M., Sohail, A., Imran, M.: Exact solutions for unsteady axial Couette flow of a fractional Maxwell fluid due to an accelerated shear. Nonlinear Anal. 16, 135-151 (2011)

16. Fetecau, C.: Analytical solutions for non-Newtonian fluid flows in pipe-like domains. Int. J. Non-Linear Mech. 39, 225-231 (2004)

17. Rubbab, Q., Husnine, S.M., Mahmood, A.: Exact solutions of generalized Oldroyd-B fluid subject to a time-dependent shear stress in a pipe. J. Prime Res. Math. 5, 139-148 (2009)

18. Kamran, M., Athar, M., Imran, M.: On the unsteady linearly accelerating flow of a fractional second grade fluid through a circular cylinder. Int. J. Nonlinear Sci. 11, 317-324 (2011)

19. Friedrich, C.H.R.: Relaxation and retardation functions of the Maxwell model with fractional derivatives. Rheologica Acta 30, 151-158 (1991)

20. Huang, J.Q., Liu, C.Q.: Analysis of general second-grade fluid flow in double cylinder rheometer. Sci. China Ser. A 26, 912-920 (1996)

21. Tan, W.C., Xian, F., Wei, L.: Exact solution for the unsteady Couette flow of the generalized second grade fluid. Chin. Sci. Bull. 47, 1226-1228 (2002)

22. Tong, D., Wang, R., Yang, H.: Exact solutions for the flow of non-Newtonian fluid with fractional derivative in an annular pipe. Sci. China Ser. G Phys. Mech. Astron. 48, 485-495 (2005)

23. Tong, D., Liu, Y.: Exact solutions for the unsteady rotational flow of non-Newtonian fluid in an annular pipe. Int. J. Eng. Sci. 43, 281-289 (2005)

24. Fetecau, C., Awan, A.U., Fetecau, C.: Taylor-Couette flow of an Oldroyd-B fluid in a circular cylinder subject to a time-dependent rotation. Bull. Math. Soc. Sci. Math. Roum. 52, 117-128 (2009)

25. Fetecau, C., Fetecau, C., Imran, M.: Axial Couette flow of an Oldroyd-B fluid due to a time dependent shear stress. Math. Rep. 11, 145-154 (2009)

26. Nazar, M., Fetecau, C., Awan, A.U.: A note on the unsteady flow of a generalized second-grade fluid through a circular cylinder subject to a time dependent shear stress. Nonlinear Anal. 11, 2207-2214 (2010)

27. Siddique, I., Vieru, D.: Exact solution for the longitudinal flow of a generalized second grade fluid in a circular cylinder. Acta Mech. Sin. 25, 777-785 (2009)

28. Wang, S., Xu, M.: Axial Couette flow of two kinds of fractional viscoelastic fluids in an annulus. Nonlinear Anal. 10, 1087-1096 (2009)

29. Qi, H., Jin, H.: Unsteady helical flow of a generalized Oldroyd-B fluid with fractional derivative. Nonlinear Anal. 10, 2700-2708 (2009)

30. Jamil, M., Fetecau, C.: Corina Fetecau, unsteady flow of viscoelastic fluid between two cylinders using fractional Maxwell model. Acta Mech. Sin. 28, 274-280 (2012)

31. Podlubny, I.: Fractional Differential Equations. Academic Press, San Diego (1999)

32. Lorenzo, C.F., Hartley, T.T.: Generalized functions for the fractional calculus, NASA/TP-1999209424/REV1 (1999) 